\title{
1 Lanthipeptide Synthetases Participate the Biosynthesis of 2-Aminovinyl-Cysteine Motifs in Thio-
}

\section{2 amitides}

3 Jingxia Lu, ${ }^{1}$ Yuan $\mathrm{Wu},{ }^{1}$ Jiao Li, ${ }^{2}$ Yuqing Li, ${ }^{1}$ Yingying Zhang, ${ }^{1}$ Zengbing Bai, ${ }^{1}$ Jie Zheng, ${ }^{1}$ Jiapeng Zhu ${ }^{2 *}$ and Huan Wang ${ }^{1}$ *

$4 \quad{ }^{1}$ State Key Laboratory of Coordination Chemistry, Chemistry and Biomedicine Innovation Center of Nanjing University,

5 Jiangsu Key Laboratory of Advanced Organic Materials, School of Chemistry and Chemical Engineering, Nanjing Universi-

6 ty, Nanjing 210093, China

$7 \quad{ }^{2}$ School of Medicine and Life Sciences, State Key Laboratory Cultivation Base for TCM Quality and Efficacy, Jiangsu Key

8 Laboratory for Pharmacology and Safety Evaluation of Chinese Materia Medica, Nanjing University of Chinese Medicine,

$9 \quad$ Nanjing 210023, China

11 Supporting Information Placeholder

ABSTRACT: Thioamitides are a group of ribosomally synthesized and post-translational modified peptides with potent antiproliferative and pro-apoptotic activities. Their biosynthesis remains largely unknown, especially for the characteristic C-

14 terminal 2-aminovinyl-Cysteine (AviCys) motifs. Herein, we report the discovery that homologs of class III lanthipeptide 15 synthetases $\left(\mathrm{LanKC}_{\mathrm{t}} \mathrm{s}\right)$ encoded outside putative thioamitide biosynthetic gene clusters (BGCs) fully dehydrate the precursor 16 peptides. Remarkably, LanKC $_{\mathrm{t}}$ enzymes bind tightly to cysteine decarboxylases encoded inside thioamitide BGCs, and the 17 resulting complex complete the macrocyclization of AviCys rings. Furthermore, LanKC $_{t}$ enzymes are present in the genomes 18 of many thioamitide-producing strains and are functional when in complex with cysteine decarboxylases to produce AviCys 19 macrocycles. Thus, our study reveals the participation of lanthipeptide synthetases as a general strategy for dehydration and 20 AviCys formation during thioamitides biosynthesis and thus paves the way for the bioengineering of this class of bioactive 21 natural products.

23 Ribosomally synthesized and post-translationally modified peptides (RiPPs) have emerged as a major family of natural 24 products with diverse bioactivities. ${ }^{1}$ Thioamitides ${ }^{2}$ are a subgroup of RiPPs that contain thioamides in place of amides in 25 peptide backbones as their class-defining feature. ${ }^{3-7}$ As the first example of this group of compounds, apoptosis inducer thi- 
oviridamide contains a number of unnatural amino acids, including backbone thioamides, a $\beta$-hydroxy-N1,N3-

dimethylhistidinium (hdmHis) residue, a N-terminal $\delta$-hydroxy- $\delta$-methyl-4-oxopentanoyl group and a C-terminal 2aminovinyl-cysteine (AviCys) motif (Fig. 1A) ${ }^{8-10}$ Posttranslational modification enzymes are identified in the $t v a$ gene cluster (Fig. S1A), including TvaH/TvaI as a pair of homologs to YcaO/TfuA proteins for the thioamidation of peptide backbone and TvaG as a putative methyltransferase for the double methylation of His(12) residue. ${ }^{11}$ The N-terminal 2-hydroxy-2methyl-4-oxopentanoyl group is the product of acetone addition to a pyruvate motif derived from the hydrolysis of a dehydroalanine (Dha) residue during purification. ${ }^{6}, 12$ The C-terminal 2-aminovinyl-cysteine (AviCys) motif is another structural feature of thioamitides and exists in a variety of RiPPs, including lanthipeptides (e.g., epidermin), ${ }^{13-15}$ lipolanthines (e.g., microvionin $)^{16}$ and linaridins (e.g., cypemycin) ${ }^{17}$ (Fig. 1A). All AviCys-containing compounds reported to date exhibit potent bioactivities, implying the biological importance of this cyclic scaffold. Current proposal for the formation of AviCys and 2-aminovinyl-3-methyl-Cysteine (AviMeCys) motifs in thioamitides involves three consecutive enzymatic modifications. First, Ser/Thr residues in the precursor peptide are converted into Dha/Dhb residues by a dehydratase. A cysteine decarboxylase then oxidatively decarboxylated the C-terminal cysteine to generate a thioenol motif. In the final step, a putative cyclase catalyzes the Michael-type addition between the thioenol group and a Dha/Dhb residue to yield an AviCys/AviMeCys motif (Fig. 1B). ${ }^{17,} 18$ Although the function of cysteine decarboxylases has been well characterized in several cases, ${ }^{19-21}$ putative dehydratases and cyclases are not yet identified in the biosynthetic gene clusters (BGCs) of thioamitides, leaving the AviCys biosynthesis unresolved. Herein, we report the discovery that homologs of class III lanthipeptide synthetases $\left(\mathrm{LanKC}_{t} \mathrm{~s}\right)$ locating outside the putative thioamitide BGCs function as dehydratases to dehydrate Ser/Thr residues in thioamitide precursor peptides. Remarkably, these LanKC $_{t}$ enzymes bind tightly to cysteine decarboxylases $\left(\mathrm{LanD}_{\mathrm{t}}\right.$, LanD-like enzymes from thioamitide biosynthesis) encoded inside thioamitide BGCs and cooperatively catalyze the formation of AviCys/AviMeCys motifs at the C-terminus of peptide substrates. The combination of $\operatorname{LanKC}_{\mathrm{t}}-\mathrm{LanD}_{\mathrm{t}}$ displays remarkable tolerance toward peptide substrates by generating AviCys/AviMeCys rings of various sizes and sequences. This study reveals an unprecedented example that lanthipeptide synthetases encoded outside thioamitide BGCs participate the macrocyclization of AviCys/AviMeCys rings through direct association with corresponding $\operatorname{LanD}_{\mathrm{t}}$ enzymes. 
A) AviCys-containing peptide natural products
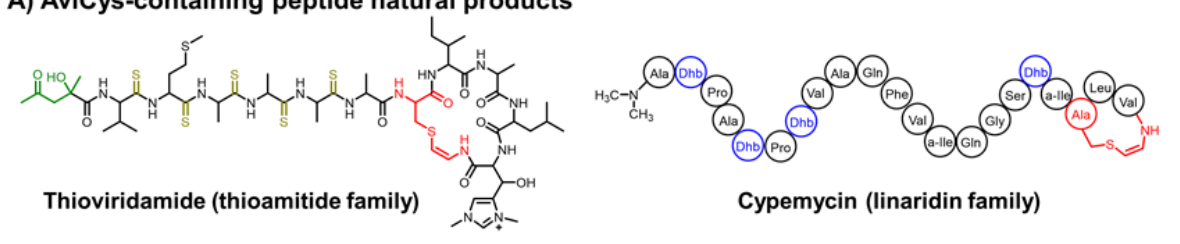

Cypemycin (linaridin family)

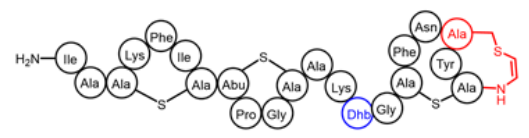

Epidermin (lanthipeptide family)

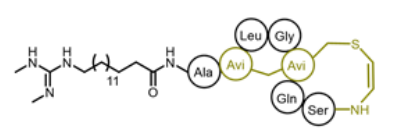

Microvionin (Lipolanthine family)
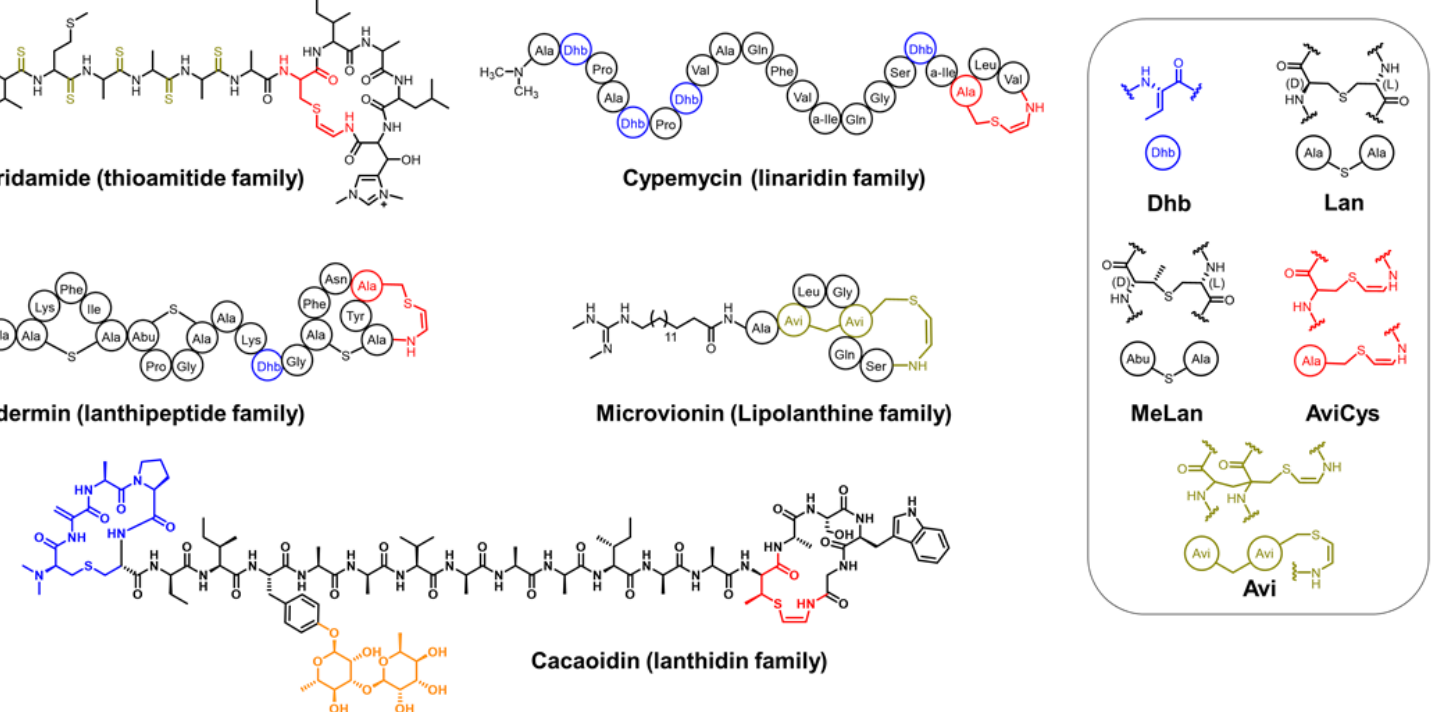

B) Proposed formation of AviCys/AviMeCys motifs

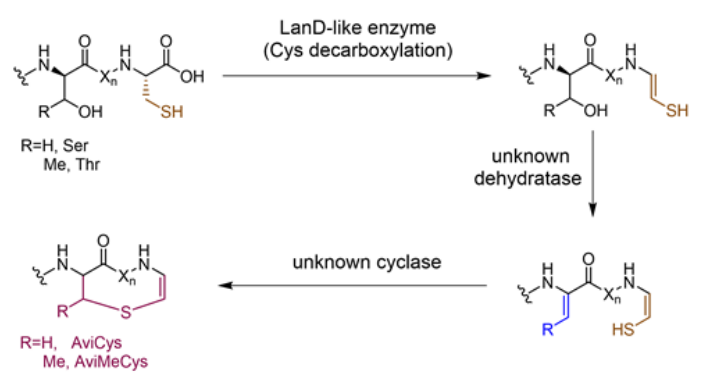

C) Biosynthesis of thiospasoamide from S. sparsogenes ATCC 25498

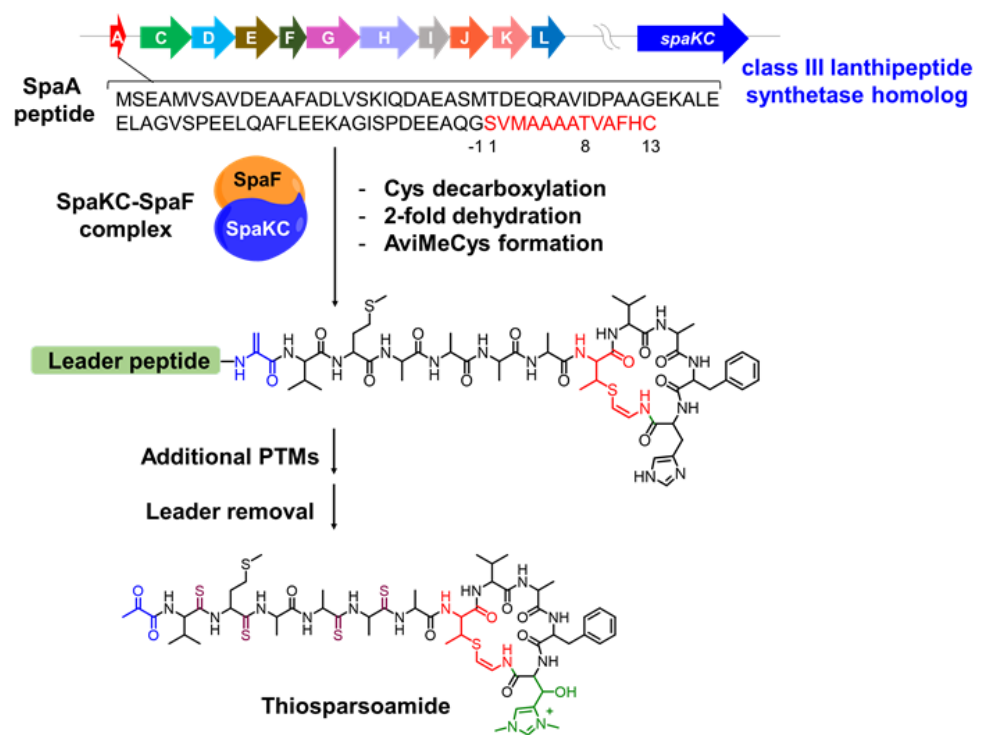

Figure 1. Structures and biosynthesis of AviCys/AviMeCys-containing compounds. A). AviCys-containing peptide natural products. B). Proposed biosynthesis of AviCys/AviMeCys motifs. C). Biosynthesis of thiosparsoamide, the putative product of the spa gene cluster. SpaA core peptide is marked in red.

\section{RESULTS}

Discovery of a new thioamitide natural product thiosparsoamide. To investigate the biosynthesis of thioamitides, we focused on a spa gene cluster from the strain of S. sparsogenes ATCC 25498, which shares high homology to the thioviridamide BGC (tva gene cluster) (Fig. 1C, Fig. S1-S2). We speculated that the final product of spa gene cluster, named thio- 
60 sparsoamide, contains multiple thioamide motifs, an hdmHis residue, a C-terminal AviMeCys macrocycle and an N-terminal

61 Dha residue, which could be hydrolyzed to a pyruvate motif in culture medium (Fig. 1C, Fig. S1). Indeed, fermentation of $S$.

62 sparsogenes strain led to the isolation of a peptide product with a mass of 1305.493 Da (Fig. S3), which matches with pre-

63 dicted mass of thiosparsoamide. Tandem MS analysis further confirmed the presence of expected unnatural amino acids in

64 this peptide product (Fig. S3). Together, these results confirm the function of spa gene cluster in producing a new thioam-

65 itide compound thiosparsoamide. 
A) Cys decarboxylation of SpaA by SpaF

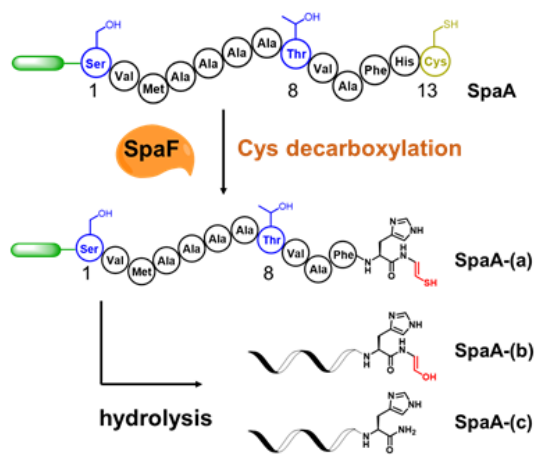

B) Crystal structure of SpaF as a homododecamer

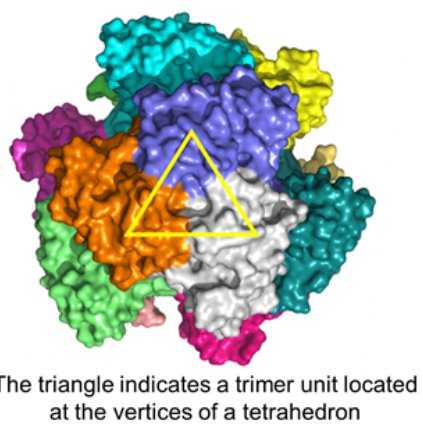

C) SpaKC fully dehydrates SpaA

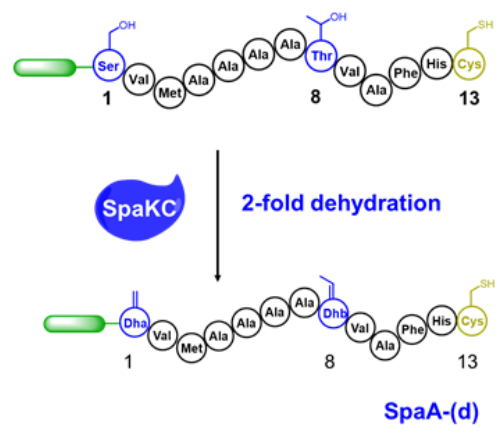

$\hookrightarrow-$ Leader peptide
D) SpaKC-SpaF installs an AviMeCys motif

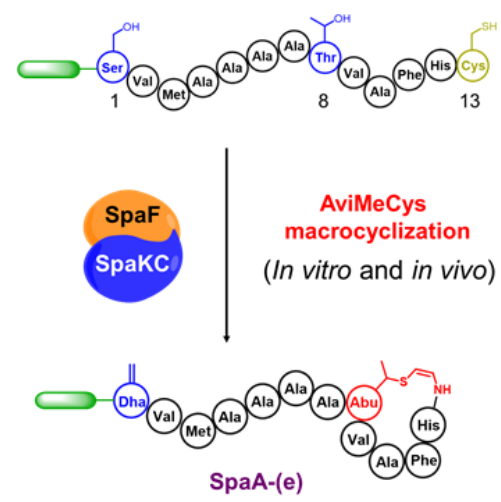

E) MS spectrum of SpaA peptide modified SpaKC and SpaF after trypsin digestion

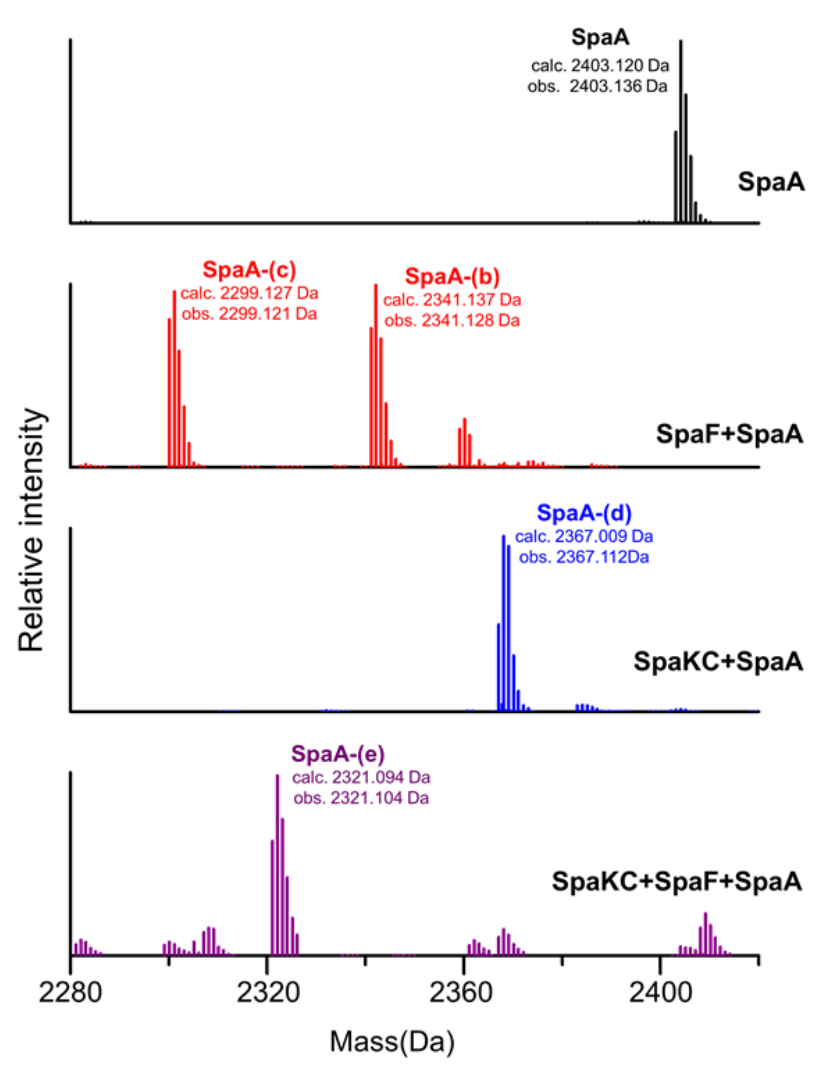

67 Figure 2. Enzymatic modification of SpaA peptide by SpaF and SpaKC via a co-expression system in E. coli. (A). SpaF

68 converts SpaA into decarboxylation product SpaA-(a), which spontaneously hydrolyzes into SpaA-(b) and SpaA-(c) in 69 buffer. (B) Crystal structure of SpaF as a homododecamer (PDB ID: 7CFU). (C) LC-MS analysis of the SpaKC-modified 70 SpaA $_{\mathrm{G}-1 \mathrm{~K}}$ peptide SpaA-(d) with 2-fold dehydration. $\operatorname{SpaA}_{\mathbf{G}-1 \mathrm{~K}}-(\mathbf{d})_{(-11)-13)}$ is the core peptide fragment of peptide $\operatorname{SpaA}_{\mathbf{G}-1 \mathrm{~K}}-(\mathbf{d})$ 
71 after trypsin digestion. (D) SpaKC-SpaF combination converts $\mathrm{SpaA}_{\mathrm{G}-1 \mathrm{~K}}$ peptide into SpaA-(e) with a Dha residue and an AviCys motif. (E) MS spectrum of modified SpaA peptides after trypsin digestion.

Class III lanthipeptide synthetase SpaKC and decarboxylase SpaF catalyze the formation of AviMeCys motif. To understand the biosynthesis of thiosparsoamide, SpaF, a putative cysteine decarboxylase encoded in the spa gene cluster, was co-expressed with precursor peptide SpaA in E. coli. SpaF oxidatively decarboxylates SpaA by producing an unstable thioenol product SpaA-(a) with a mass loss of 46 Da (Fig. 2A, Fig. S4). SpaA-(b) and SpaA-(c) with mass losses of 62 Da and $104 \mathrm{Da}$, respectively, were detected as the hydrolysis products of SpaA-(a) (Fig. 2A, Fig. S4-S5). Degradation of thioenol products from peptide decarboxylation is spontaneous in buffer conditions and precedent in the cases of several LanD-like enzymes, including TvaF from thioviridamide biosynthesis, EpiD from epidermin biosynthesis and MicD from microvionin biosynthesis. ${ }^{16,22}$ To facilitate the removal of leader peptide, $\mathrm{SpaA}_{\mathrm{G}-1 \mathrm{~K}}$ peptide was used as peptide substrate for further studies (Fig. S6-S7). To provide mechanistic insight into the function of SpaF, we resolved its crystal structure at $2.15 \AA$ resolution. In solution, SpaF forms a complex with a molecular weight $\sim 300 \mathrm{kDa}$ with FMN bound as a cofactor (Fig. S8). Consistent with this observation, crystal-packing shows that SpaF assembles into a homododecamer, in which trimers locate at the vertices of a tetrahedron (Fig. 2B). Each trimer unit binds with one FMN molecule in a cavity at the interface of two monomers (Fig. S9). The overall structure of SpaF is highly similar to those of TvaF, CypD and EpiD despite their low sequence similarity, suggesting the convergent evolution of cysteine decarboxylases of this class. ${ }^{23}$ Together, our results showed that SpaF is a FMN-dependent enzyme that catalyzes the cysteine decarboxylation of the precursor peptide SpaA.

Next, we focused on elucidating the identity of dehydratase and cyclase for the dehydration and AviMeCys formation in thiosparsoamide biosynthesis (Fig. 1C). We noticed that leader peptides of thioamitide precursors contain highly conserved PEE(L/A)Q motifs, which shares similarity to the LELQG motif conserved in leader peptides of class III lanthipeptide precursors for enzymatic recognition (Fig. S2 and S10). ${ }^{24,25}$ In addition, class III lanthipeptide synthetase (LanKC) AplKC from NAI-112 biosynthesis is able to phosphorylate SpaA peptide in vitro (Fig. S11), indicating that SpaA can be recognized by a LanKC enzyme. ${ }^{26,27}$ Together, these data raise the possibility that SpaA peptide could be modified by a LanKC enzyme during thiosparsoamide biosynthesis. By scanning the genome of S. sparsogenes ATCC 25498, we found a gene, named spaKC, which encodes a LanKC homolog and is located far outside the spa gene cluster without association with any putative biosynthetic gene clusters of secondary metabolites (Fig. 1C, Table S1). Co-expression of $s p a K C$ and $s p a A_{G-I K}$ genes produced $\mathbf{S p a A}_{\mathbf{G - 1 K}}-\mathbf{( d )}$ with 2-fold dehydration at $\operatorname{Ser}(1)$ and $\operatorname{Thr}(8)$ residues as the only product, showing that SpaKC efficiently recognizes $\mathrm{SpaA}_{\mathrm{G}-1 \mathrm{~K}}$ as a substrate for dehydration (Fig. 2B, Fig. S12). No ring structure was formed in $\mathbf{S p a A}_{\mathbf{G}-1 K^{-}}$(d), suggest- 
100 ing that SpaKC is not capable of catalyzing the formation of a Lan/MeLan macrocycle between Cys(13) and $\mathrm{Dha}(1) / \mathrm{Dhb}(8)$

101 or $\operatorname{SpaA}_{\mathbf{G - 1 K}}-\mathbf{( d )}$ is not a substrate for macrocyclization. Next, $\mathrm{SpaA}_{\mathrm{G}-1 \mathrm{~K}}$ peptide was co-expressed with SpaKC and SpaF

102 enzymes in E. coli, yielding a peptide product $\mathbf{S p a A}_{\mathbf{G}-1 \mathbf{K}}-(\mathbf{e})$ with a mass loss of 82 Da compared with SpaA peptide, match103 ing two-fold dehydration (-36 Da) and one oxidative decarboxylation (-46 Da) (Fig. 2C). Tandem MS analysis showed that

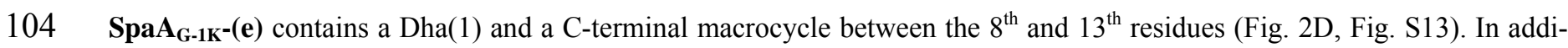
105 tion, iodoacetamide (IAA) treatment of $\mathbf{S p a A}_{\mathbf{G}_{-1} \mathbf{K}^{-}} \mathbf{( e )}$ resulted in no mass change, supporting that the thiol group of Cys(13) 106 was consumed during the ring formation (Fig. S14).

107 To further verify the identity of the C-terminal macrocycle, SpaA $\mathbf{A}_{\mathbf{G}-\mathbf{K}} \mathbf{-}(\mathbf{e})$ peptide was reduced and desulfurized by $\mathrm{NiCl}_{2}$ and $108 \mathrm{NaBH}_{4}$ treatment in $\mathrm{H}_{2} \mathrm{O}$ following an established protocol (Fig. 3A, Fig. S15). ${ }^{28}$ Tandem MS analysis of the desulfurization 109 product $\mathbf{S p a A}_{\mathbf{G}-\mathbf{1 K}}-\mathbf{( f )}$ revealed the presence of an Ala(1) residue, two homoalanine (hAla) residues at the $3^{\text {rd }}$ and $8^{\text {th }}$ positions 110 and an ethylamine-derived His residue at the C-terminus, supporting the structural assignment of $\mathbf{S p a A}_{\mathbf{G}-1 \mathbf{K}^{-}}(\mathbf{e})$ with a Dha(1)

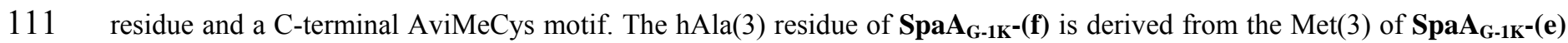
112 via sidechain cleavage during desulfurization. When $\mathrm{NaBD}_{4}$ and $\mathrm{D}_{2} \mathrm{O}$ were employed to linearize $\mathbf{S p a A}_{\mathbf{G}-1 K^{-}}(\mathbf{e})$, Dha(1) was 113 reduced to an Ala residue with double deuteration, whereas Met(8) was converted into a hAla residue with one deuterium 114 substitution in product $\mathbf{S p a A}_{(\mathrm{G}-1 \mathrm{~K})}$-(g) (Fig. 3B, Fig. S16). Importantly, single deuterium and triple deuterium substitutions 115 were observed in the $\mathrm{hAla}(8)$ residue and the C-terminal $12^{\text {th }}$ residue in $\mathbf{S p a A} \mathbf{A}_{(\mathrm{G}-1 \mathrm{~K})}-\mathbf{( g )}$, respectively, further supporting the 116 installation of a C-terminal AviMeCys motif in SpaA peptide. Collectively, our results showed that the combination of SpaF117 SpaKC enzymes is capable of catalyzing the AviMeCys formation in precursor peptide SpaA. 


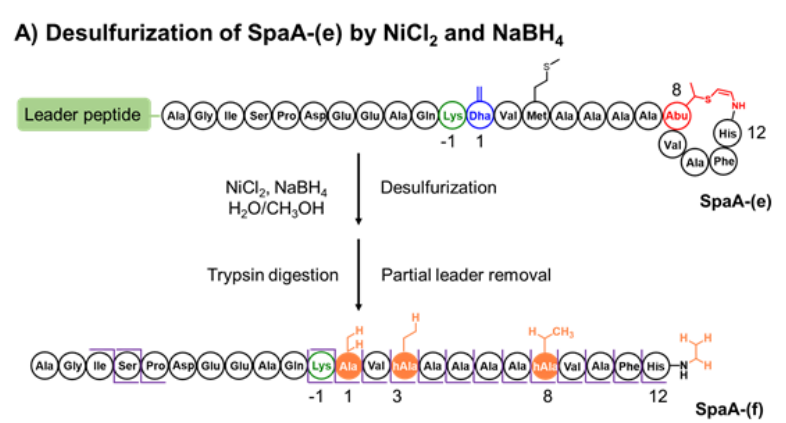

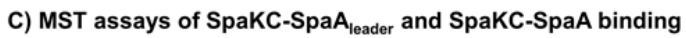

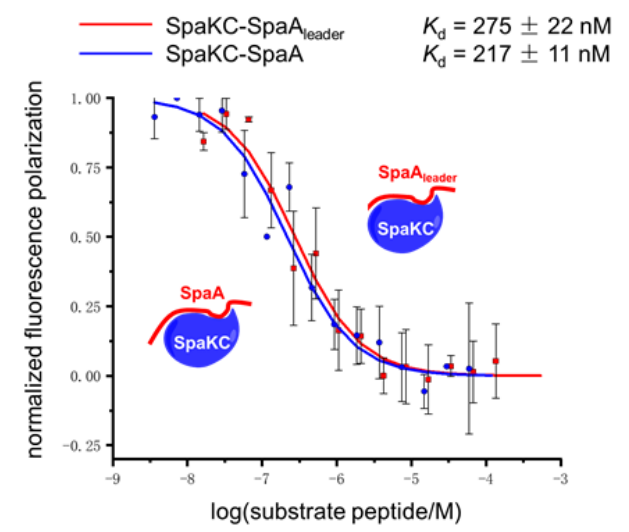

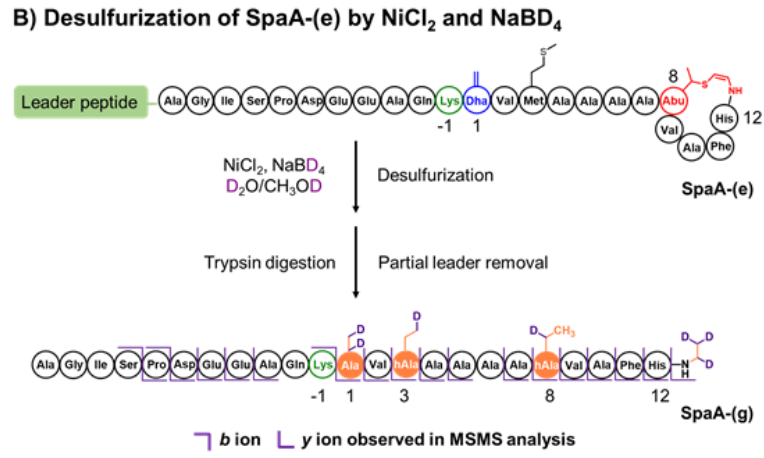

D) MST assays of SpaKC-SpaF and SpaKC-SpaF-SpaA binding

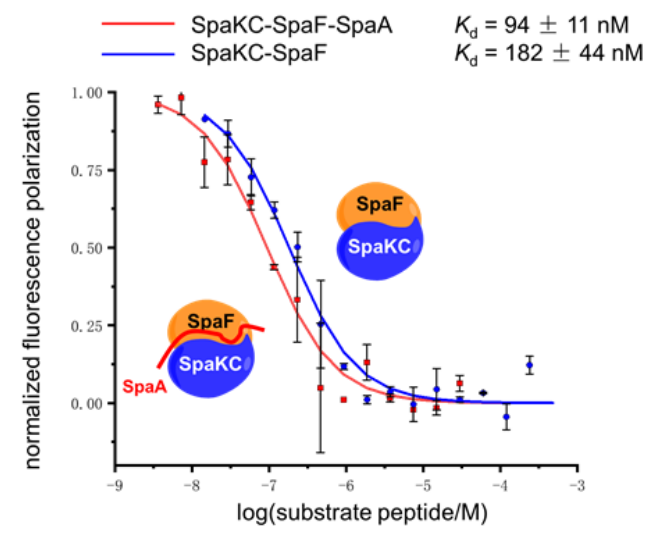

119 Figure 3. Characterization of AviMeCys formation and binding affinities between SpaKC, SpaF and SpaA. (A-B) 120 Characterization of AviMeCys formation in SpaA-(e) peptide by reductive desulferization and MS/MS analysis of the 121 resulting linearized product. $b$ ions and $y$ ions resulted from fragmentation are shown in purple. (C-D) MST analysis of the 122 binding affinity between SpaKC and SpaA, SpaA leader peptide, SpaF. Error bars, s.e.m. of three independent measurements. 123 Experimental details are described in the supporting information.

125 SpaKC and SpaF function as a complex. The formation of AviMeCys motif includes dehydration of Ser/Thr residues, cys126 teine decarboxylation and AviMeCys macrocyclization. To probe the order of modifications, we examined the in vitro activi127 ty of SpaKC and SpaF toward various peptide substrates. SpaKC is fully functional as a dehydratase in vitro with ATP as a 128 cofactor to dehydrate SpaA and decarboxylated SpaA peptides (Fig. S17-S18). Similarly, SpaF is highly efficient in decar-

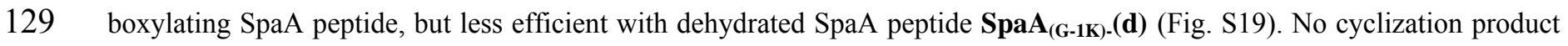
130 was detected in the abovementioned assays when SpaKC or SpaF functions alone. Only when SpaA, SpaKC and SpaF were 131 incubated together, cyclized product $\mathbf{S p a A}_{\mathbf{G}-1 \mathbf{K}^{-}}(\mathbf{e})$ was successfully produced in vitro (Fig. 2D, Fig. S20), indicating that the 132 combination of SpaKC and SpaF enzymes was strictly required for AviMeCys installation. In order to understand the coop133 erative nature of SpaKC-SpaF enzymes, the interactions between SpaKC, SpaF and SpaA peptides were measured by mi- 
134 croscale thermophoresis (MST) assays (Fig. 3C and 3D). Results showed that SpaKC binds to SpaA leader peptide and SpaA 135 peptide with similar $K_{\mathrm{d}}$ values of $(275 \pm 23) \mathrm{nM}$ and $(217 \pm 12) \mathrm{nM}$, indicating that SpaKC functions in a leader dependent 136 manner. Remarkably, SpaKC and SpaF bind to each other tightly with a $K_{\mathrm{d}}$ of $(182 \pm 44) \mathrm{nM}$, showing that SpaKC and SpaF 137 function as a complex in solution. Remarkably, the binding between SpaKC-SpaF complex and SpaA peptide exhibited a $K_{\mathrm{d}}$ 138 of $(94 \pm 11) \mathrm{nM}$, which is 2-fold lower than that of SpaKC-SpaA binding, indicating that SpaA peptide enhances the stability 139 of the enzyme complex during modification. Together, our results show that SpaKC and SpaF function as a complex and 140 process peptide substrate in a leader dependent manner to achieve AviMeCys macrocyclization (Fig. 4).
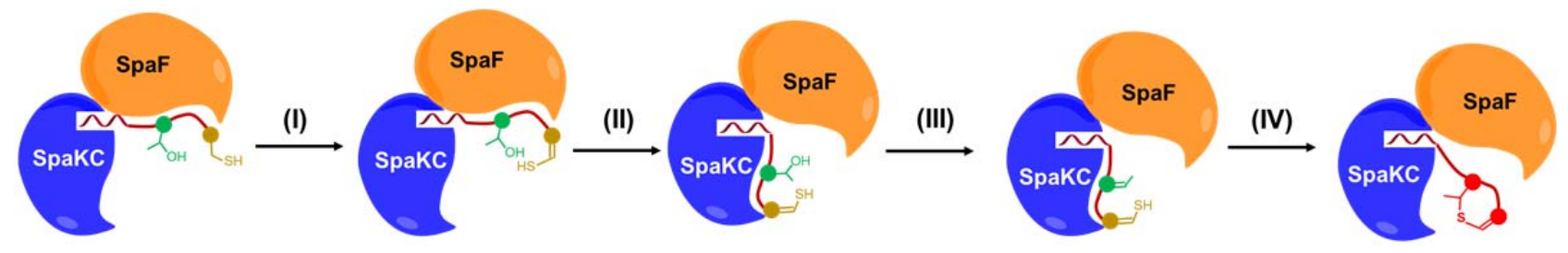

142 Figure 4. Current model of AviMeCys formation catalyzed by SpaKC-SpaF complex by the following steps: (I). First, SpaA 143 peptide binds to the SpaKC-SpaF complex via leader-SpaKC association; (II). As the first modification, SpaF oxidatively 144 decarboxylates the C-terminal Cys residue in SpaA; (III). The decarboxylated core peptide is relocated to SpaKC for dehydration; (IV). The dehydrated and decarboxylated SpaA peptide is cyclized to form an AviMeCys motif.

Next, we examined the versatility of SpaKC-SpaF complex in generating AviCys/AviMeCys motifs in various SpaA mutants. Mutation of AviMeCys precursor residues $\operatorname{Thr}(8)$ and Cys(13) to Cys and Ala, respectively, abolished the AviMeCys formation while dehydration of Ser/Thr occurs efficiently (Fig. 5A, entry 1-2, Fig. S21-22). Ala mutation at any other position of SpaA, including residues in the ring region, has little impact on the macrocyclization of AviMeCys motif in the corresponding SpaA peptide (Fig. 5A, entry 3-5, Fig. S23-25). Furthermore, SpaKC-SpaF combination exhibited remarkable flexibility when the size of the AviMeCys ring was altered. Deletion or insertion of residues between $\operatorname{Thr}(8)$ and Cys(13) did not significantly affect macrocyclization, and AviMeCys rings of sizes varying from four to eight amino acids were efficiently generated (Fig. 5B, entry 6-11, Fig. S26-31). Similarly, SpaA peptides carrying mutations or deletion of non-ring-forming residues in the core peptide were well tolerated by SpaKC-SpaF combination by yielding corresponding cyclized products (Fig. 5A, entry 12-14, Fig. S32-34). In addition, $\mathrm{SpaA}_{\mathrm{T} 8 \mathrm{~S}}$ peptide were efficiently cyclized by forming an AviCys motif, fur- 
Utilization of LanKC enzymes for dehydration and AviCys cyclization is a general strategy for thioamitide

160 biosynthesis. The thioamitide precursor peptides share high sequence similarity (Fig. S2A), raising the possibility to utilize

161 SpaKC as an enzymatic tool to dehydrate these peptides. Five precursor peptides, including TvaA from S. olivoridis

162 NA05001, TsdA from $S$. $s p$ NRRL S-87, MutA from S. mutomycini NRRL B65393, AlbA from A. alba DSM 44262, and

163 MalA(136) from S. malaysiense MUSC 136 (Fig. S2A), were co-expressed with SpaKC in E. coli. As expected, SpaKC

164 efficiently modified all five precursor peptides with two-fold dehydration (Fig. S36-40), demonstrating the substrate

165 tolerance of SpaKC as a dehydratase.

166 Next, we examined whether SpaKC could function with LanD $_{t}$ decarboxylases from thioamitide BGCs by installing 167 AviCys/AviMeCys motifs in corresponding precursor peptides. TvaF from thioviridamide biosynthesis was selected as a 168 model and shown to bind to SpaKC with a $K_{\mathrm{d}}$ of $(2.27 \pm 0.85) \mu \mathrm{M}$ (Fig. S41), indicating that SpaKC and TvaF can function 169 as a complex. When co-expressed with TvaA peptide in E. coli, SpaKC-TvaF combination efficiently modified the peptide 170 substrate by installing one Dha at the $1^{\text {st }}$ position and one AviCys motif at the C-terminus (Fig. 5B, Fig. S42). Similarly, 171 when SpaKC was co-expressed with MutF/MutA, TsdF/TsdA, MalF(136)/MalA(136) and AlbF/AlbA pairs, AviCys motifs 172 were correctly installed at the C-terminus in all peptide substrates (Fig. 5A, Fig. S43-S46). Therefore, SpaKC serves as a 173 general dehydratase and functions cooperatively with $\mathrm{LanD}_{\mathrm{t}}$ enzymes by forming protein complexes to install 174 AviCys/AviMeCys motifs in thioamitide precursor peptides.

A) Substrate tolerance of SpaKC-SpaF combination for AviCys/AviMeCys cyclization

\begin{tabular}{|c|c|c|c|}
\hline Entry & SpaA peptides ( leader peptide $=\backsim$ ) & Mutation & Avi(Me)Cys \\
\hline 1 & 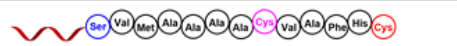 & T8C & $x$ \\
\hline 2 & 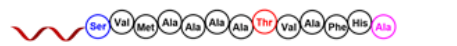 & C13A & $x$ \\
\hline 3 & V & V9A & $\checkmark$ \\
\hline 4 & 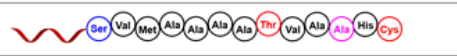 & F11A & $\checkmark$ \\
\hline 5 & 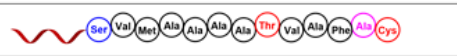 & H12A & $\checkmark$ \\
\hline 6 & 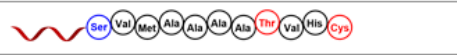 & A10\&F11 deletion & $\boldsymbol{v}$ \\
\hline 7 & 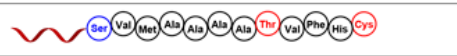 & A10 deletion & $\checkmark$ \\
\hline 8 & 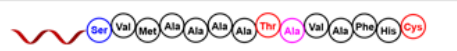 & T8insertA & $\checkmark$ \\
\hline 9 & 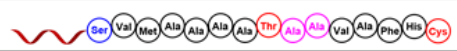 & T8insertAA & $\checkmark$ \\
\hline 10 & 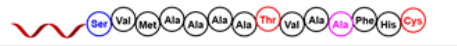 & A10insertA & $\checkmark$ \\
\hline 11 & 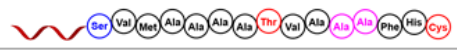 & A10insertAA & $\checkmark$ \\
\hline 12 & 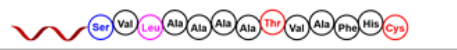 & M3L & $\boldsymbol{v}$ \\
\hline 13 & 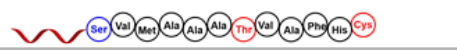 & A7 deletion & $\checkmark$ \\
\hline 14 & 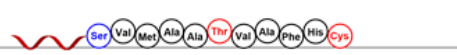 & A6\&A7 deletion & $\checkmark$ \\
\hline 15 & 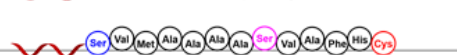 & T8S & $\checkmark$ \\
\hline
\end{tabular}

B) SpaKC-LanD, combinations cyclize thioamitide precursor peptides

\begin{tabular}{|c|c|c|c|}
\hline Precursor peptides & Enzyme(s) & Dehydration & Avi(Me)Cys \\
\hline TvaA: Leader-SVMAAAASIALHC & TvaF + SpaKC & One-fold & $\checkmark$ \\
\hline MutA & MutF + SpaKC & One-fold & $\checkmark$ \\
\hline TsdA & TsdF + SpaKC & One-fold & $\checkmark$ \\
\hline MalA(136) & MalF(136) + SpaKC & One-fold & $\checkmark$ \\
\hline AlbA & AlbF + SpaKC & One-fold & $\checkmark$ \\
\hline
\end{tabular}

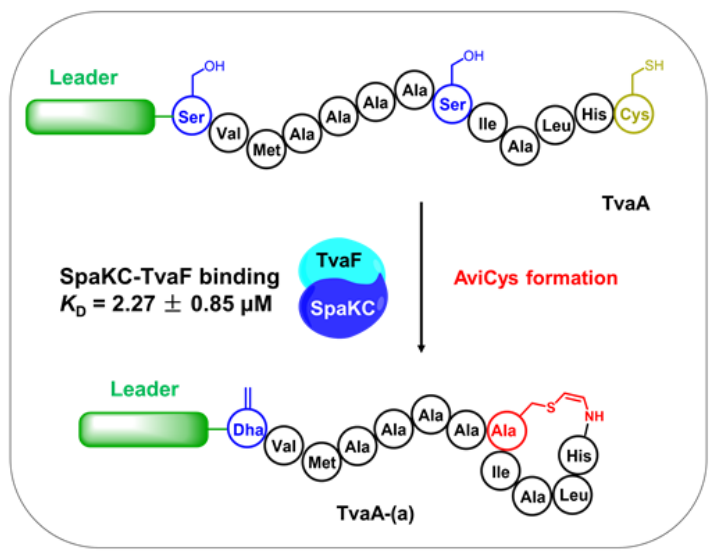


176 Figure 5. SpaKC and SpaF function as a complex and allows generation of AviCys/AviMeCys rings of various sizes and

177 sequences. SpaKC installs Dha residues and AviCys/AviMeCys motifs in precursor peptides of thioviridamide-like natural 178 products. (A). Precursor peptides of thioviridamide-like natural products are modified by SpaKC or the enzyme pair of 179 SpaKC-(cysteine decarboxylase). (B). SpaKC and TavF form a complex and modify TvaA peptide by installing a Dha 180 residue and a C-terminal AviCys motif.

182 The capability of SpaKC to function with various $\mathrm{LanD}_{\mathrm{t}}$ enzymes for AviCys/AviMeCys formation implies that the 183 utilization of class III lanthipeptide synthetase (LanKC) homologs might be a general strategy for thioamitide biosynthesis. 184 To date, 18 bacterial strains have been identified to contain putative thioamitide BGCs in their genomes. By scanning the 185 genomes of these bacteria (17 out of 18 are fully sequenced), we identified 12 genes encoding LanKC homologs from 10 186 strains, which are all located far outside the putative thioamitide BGCs (Table 1, Table S1). Both sequence Similarity Net187 works (SSNs) analysis and maximum-likehood phylogeny analysis show that these LanKC homologs (named LanKC $\mathrm{t}_{\mathrm{t}}$, 188 LanKC homologs in thioamitide biosynthesis), including SpaKC, are closely related to known class III lanthipeptide 189 synthetases phylogenetically (Fig. S47-S48). To examine the participation of LanKC $\mathrm{C}_{\mathrm{t}}$ enzymes in thioamitide biosynthesis, 190 we selected MalA(136), the precursor peptide from the thioamitide BGC in S. malaysiense MUSC 136 strain, as well as the 191 LanKC $_{\mathrm{t}}$ enzyme MalKC(136)-1 discovered from its genome, as a model (Fig. S49). Results showed that MalKC(136)-1 192 efficiently dehydrated MalA(136) peptide by up to 2-fold during co-expression in E. coli (Fig. S50), confirming the role of 193 MalKC(136)-1 as a dehydratse to modify the corresponding thioamitide precursor peptide. Together, our results indicate that 194 the participation of a LanKC $\mathrm{t}_{\mathrm{t}}$ enzymes encoded outside corresponding thioamitide BGCs is a general strategy employed 195 during thioamitide biosynthesis. 


\begin{tabular}{|c|c|c|}
\hline Strain & LanKC & Accession number \\
\hline S. sparsogenes ATCC 25498 & SpaKC & WP_065963996.1 \\
\hline S. sp. MUSC 14 & SmuKC(14) & WP_071371419.1 \\
\hline \multirow{2}{*}{ S. sp. MUSC 125 } & SmuKC(125) & WP_039650135.1 \\
\hline \multirow{2}{*}{ S. malaysiense MUSC 136 } & MalKC(136)-1 & OIK27458.1 \\
\cline { 2 - 3 } & MalKC(136)-2 & OIK27459.1 \\
\hline \multirow{2}{*}{ S. malaysiense } & MalKC & WP_071381943.1 \\
\hline \multirow{2}{*}{ S. $s p$. NRRL S-4 } & ScnKC-1 & WP_026290248.1 \\
\cline { 2 - 3 } & ScnKC-2 & WP_018959331.1 \\
\hline S. mutomycini NRRL B-65393 & SnrKC(S-4) & WP_053928640.1 \\
\hline Amycolatopsis alba DSM 44262 & AmyKC & WP_020633044.1 \\
\hline Nocardiopsis potens DSM 45234 & NocKC & WP_017595403.1 \\
\hline
\end{tabular}

197 Table 1. LanKC $\mathrm{t}_{\mathrm{t}}$ genes discovered in the genomes of thioamitide-producing strains. All genes are located outside 198 corresponding thioamitide BGCs.

\section{Discussion}

200 AviCys/AviMeCys motifs exist in several classes of RiPPs and are essential for their bioactivities. Elucidation of the 201 multiple enzymatic transformations during AviCys/AviMeCys formation is a prerequist for the bioengineeing of these 202 bioactive compounds. Our investigation reveals that LanKC $\mathrm{t}_{\mathrm{t}}$ enzymes encoded outside putative thioamitide BGCs fully 203 dehydrate the precursor peptides. LanKC $C_{t}$ and $\operatorname{LanD}_{t}$ enzymes encoded inside thioamitide BGCs may form complexes and 204 construct the AviCys/AviMeCys rings cooperatively. Regarding the order of modifications during AviCys formation, our 205 results suggest that cysteine decarboxylation occurs before the dehydration of precursor peptides. The formation of a 206 LabKC $_{t}-\operatorname{LanD}_{t}$ complex is therefore benefical for the unstable thioenol intermediate to quickly relocate from LanD $D_{t}$ to the 207 LanKC $_{t}$ enzyme for dehydration and subsequent cyclization by minimizing possibility of hydrolysis. We further show that 208 LanKC $_{t}$ enzymes are widespread in the genomes of most thioamitide-producing strains and are all functional to dehydrate 209 corresponding thioamitide precursor peptides, suggesting $\operatorname{LanKC}_{\mathrm{t}}-\mathrm{LanD}_{\mathrm{t}}$ combination is a general strategy for 210 AviCys/AviMeCys formation. Together, our study reveals a very rare system in the biosynthesis of RiPPs that a modification 211 enzyme encoded outside the BGC function cooperatively with an enzyme encoded inside the BGC through specific 212 association to construct a key structural element in the final product. The detailed catalytic mechanism for 213 AviCys/AviMeCys cyclization in LanKC $_{t}-$ LanD $_{t}$ complex requires further investigation. 
214 It is highly possible that proteins of unknown functions encoded in the putative thioamitide BGCs possess dehydratase

215 function for AviCys formation, since 8 out of 18 thioamitide-producing strains do not contain LanKC $\mathrm{t}_{\mathrm{t}}$ enzymes in their

216 genomes. In addition, successful heterologous production of thioviridamide and derivatives by the putative tva gene cluster

217 has been reported in S. lividans and S. avermitilis, suggesting the tva gene cluster is fully capable of producing

218 thioviridamides. ${ }^{10,29-31}$ However, it is noteworthy that LanKC $\mathrm{C}_{\mathrm{t}}$ enzymes are encoded in the genomes of both S. lividans and $S$.

219 avermitilis strains, and their functions remain unknown (Table S2). Therefore, we hypothesize that LanKC $\mathrm{C}_{\mathrm{t}}$ enzymes may not

220 be essential for thioamitide biosynthesis, but serve as complementary dehydratases to support the production of thioamitides

221 when neccesasry.

222 Recently, coorpoerative function of MicKC-MicD enzyme pair is reported in the formation of avionin motifs during 223 microvionin biosynthesis, in which both enzymes are encoded in the BGCs (Fig. 1A, Fig. S51). ${ }^{29}{ }^{32}$ MicKC and MicD 224 function in a mutual regulotory manner, where the activity of MicKC is highly dependent on the presence of MicD. In 225 contrast, SpakC and SpaF are fully functional saparately for dehydration and decarboxylation, respectively, desipte the 226 requirement of complex formation for AviCys/AviMeCys macrocyclization. The biosynthesis of lipolanthines and 227 thioamitides suggest that the cooperative function of LanKC- and LanD-like enzymes for AviCys-like macrocyclization 228 might be a general biosynthetic strategy, which requires further investigation to understand the detailed enzymatic 229 mechanisms.

\section{ASSOCIATED CONTENT}

\section{Supporting Information}

232 Detailed compound characterization and MS spectra are provided. Supporting Information is available free of charge via the 233 Internet at http://pubs.acs.org.

\section{AUTHOR INFORMATION}

\section{Corresponding Author}

237 *wanghuan@nju.edu.cn

\section{Author Contributions}

239 The manuscript was written through contributions of all authors. All authors have given approval to the final version of the 240 manuscript. 


\section{Notes}

243 The authors declare no competing financial interests.

\section{ACKNOWLEDGMENT}

This work is supported by NSF of China (Grant 21778030 and 21861142005 to H.W.) and the Fundamental Research Funds

for the Central Universities (Grant 14380138 and 14380131 to H.W.).

\section{REFERENCES}

1. Arnison, P.G. et al. Ribosomally synthesized and post-translationally modified peptide natural products: overview and recommendations for a universal nomenclature. Nat. Prod. Rep. 30, 108-160 (2013).

2. Note: The term thioamitides is suggested to describe this family of compounds that have thioamides as the classdefining feature. These compounds are referred as thioviridamide-like compounds or polythioamides in previous literatures.

3. Tang, J., Lu, J.X., Luo, Q.F. \& Wang, H. Discovery and biosynthesis of thioviridamide-like compounds. Chinese Chem. Lett. 29, 1022-1028 (2018).

4. Frattaruolo, L. et al. Thioalbamide, A Thioamidated Peptide from Amycolatopsis alba, Affects Tumor Growth and Stemness by Inducing Metabolic Dysfunction and Oxidative Stress. Cells 8, 1408 (2019).

5. Takase, S. et al. Mechanism of Action of Prethioviridamide, an Anticancer Ribosomally Synthesized and PostTranslationally Modified Peptide with a Polythioamide Structure. ACS Chem. Biol. 14, 1819-1828 (2019).

6. $\quad$ Frattaruolo, L., Lacret, R., Cappello, A.R. \& Truman, A.W. A Genomics-Based Approach Identifies a Thioviridamide-Like Compound with Selective Anticancer Activity. ACS Chem. Biol. 12, 2815-2822 (2017).

7. Kjaerulff, L. et al. Thioholgamides: Thioamide-Containing Cytotoxic RiPP Natural Products. ACS Chem. Biol. 12, 2837-2841 (2017).

8. Hayakawa, Y. et al. Thioviridamide, a novel apoptosis inducer in transformed cells from Streptomyces olivoviridis. J. Antibiot. 59, 1-5 (2006).

9. Hayakawa, Y., Sasaki, K., Nagai, K., Shin-ya, K. \& Furihata, K. Structure of thioviridamide, a novel apoptosis inducer from Streptomyces olivoviridis. J. Antibiot. 59, 6-10 (2006).

10. Izawa, M., Kawasaki, T. \& Hayakawa, Y. Cloning and Heterologous Expression of the Thioviridamide Biosynthesis Gene Cluster from Streptomyces olivoviridis. Appl. Environ. Microbiol. 79, 7110-7113 (2013).

11. Mahanta, N., Liu, A., Dong, S., Nair, S.K. \& Mitchell, D.A. Enzymatic reconstitution of ribosomal peptide backbone thioamidation. Proc. Natl. Acad. Sci. U S A 115, 3030-3035 (2018).

12. Izawa, M., Nagamine, S., Aoki, H. \& Hayakawa, Y. Identification of essential biosynthetic genes and a true biosynthetic product for thioviridamide. J. Gen. Appl. Microbiol. 64, 50-53 (2018).

13. Novak, J., Caufield, P.W. \& Miller, E.J. Isolation and biochemical characterization of a novel lantibiotic mutacin from Streptococcus mutans. J. Bacteriol. 176, 4316-4320 (1994).

14. Schnell, N. et al. Prepeptide sequence of epidermin, a ribosomally synthesized antibiotic with four sulphide-rings. Nature 333, 276-278 (1988).

15. Tao, M. et al. Functional genome mining reveals a novel class V lanthipeptide containing a D-amino acid introduced by an F420H2-dependent reductase. Angew. Chem. Int. Ed., DOI: 10.1002/anie.202008035 (2020).

16. Wiebach, V. et al. The anti-staphylococcal lipolanthines are ribosomally synthesized lipopeptides. Nat. Chem. Biol. 14, 652-654 (2018).

17. Sit, C.S., Yoganathan, S. \& Vederas, J.C. Biosynthesis of aminovinyl-cysteine-containing peptides and its application in the production of potential drug candidates. Acc. Chem. Res. 44, 261-268 (2011).

18. Truman, A.W. Cyclisation mechanisms in the biosynthesis of ribosomally synthesised and post-translationally modified peptides. Beilstein J. Org. Chem. 12, 1250-1268 (2016).

19. Ding, W., Mo, T., Mandalapu, D. \& Zhang, Q. Substrate specificity of the cypemycin decarboxylase CypD. Synth. Syst. Biotechnol. 3, 159-162 (2018).

20. Lu, J. et al. Characterization of the FMN-Dependent Cysteine Decarboxylase from Thioviridamide Biosynthesis. Org. Lett. 21, 4676-4679 (2019). 
21. Ding, W. et al. Cypemycin Decarboxylase CypD Is Not Responsible for Aminovinyl-Cysteine (AviCys) Ring Formation. Org. Lett. 20, 7670-7673 (2018).

22. Kupke, T., Kempter, C., Jung, G. \& Gotz, F. Oxidative decarboxylation of peptides catalyzed by flavoprotein EpiD. Determination of substrate specificity using peptide libraries and neutral loss mass spectrometry. J. Biol. Chem. 270, 11282-11289 (1995).

23. Mo, T.L. et al. Convergent evolution of the Cys decarboxylases involved in aminovinyl-cysteine (AviCys) biosynthesis. FEBS Lett. 593, 573-580 (2019).

24. Wang, H. \& van der Donk, W.A. Biosynthesis of the class III lantipeptide catenulipeptin. ACS Chem. Biol. 7, 15291535 (2012).

25. Muller, W.M., Ensle, P., Krawczyk, B. \& Sussmuth, R.D. Leader peptide-directed processing of labyrinthopeptin A2 precursor peptide by the modifying enzyme LabKC. Biochemistry 50, 8362-8373 (2011).

26. Chen, S. et al. Zn-dependent bifunctional proteases are responsible for leader peptide processing of class III lanthipeptides. Proc. Natl. Acad. Sci. U S A 116, 2533-2538 (2019).

27. Iorio, M. et al. A glycosylated, labionin-containing lanthipeptide with marked antinociceptive activity. ACS Chem. Biol. 9, 398-404 (2014).

28. Lohans, C.T. et al. Structural characterization of the highly cyclized lantibiotic paenicidin A via a partial desulfurization/reduction strategy. J. Am. Chem. Soc. 134, 19540-19543 (2012).

29. Zheng, X. et al. Condensation of 2-((Alkylthio)(aryl)methylene)malononitrile with 1,2-Aminothiol as a Novel Bioorthogonal Reaction for Site-Specific Protein Modification and Peptide Cyclization. J. Am. Chem. Soc. 142, 5097-5103 (2020).

30. Izumikawa, M. et al. Novel thioviridamide derivative-JBIR-140: heterologous expression of the gene cluster for thioviridamide biosynthesis. J. Antibiot. 68, 533-536 (2015).

31. Kudo, K. et al. Comprehensive Derivatization of Thioviridamides by Heterologous Expression. ACS Chem. Biol. 14, 1135-1140 (2019).

32. Wiebach, V. et al. An amphipathic alpha-helix guides maturation of the ribosomally-synthesized lipolanthines. Angew. Chem. Int. Ed., DOI: 10.1002/anie.202003804 (2020). 
General methods. Primers, genes and peptide were synthesized by Genscript Biotech (Nanjing, China). Restriction endonucleases and T4 DNA ligase were purchased from New England Biolabs (Ipswich, MA, USA). Phanta ${ }^{\circledR}$ Max Master Mix were purchased from Vazyme Biotech Co., Ltd (Nanjing, China). Medium components for bacterial cultures were purchased from Thermo Fisher (Waltham, MA, USA). Chemicals were purchased from Aladdin Reagent (Shanghai, China) and SigmaAldrich (Schnelldorf, Germany). Endoprotease trypsin was purchased from Roche Biosciences (Basel, Switzerland). E. coli DH5 $\alpha$ was used as a host for cloning and plasmid propagation, and E. coli BL21 (DE3) was used as a host for expression of proteins and peptides. S. sparsogenes ATCC 25498 is purchased from (China General Microbiological Culture Collection Center, CGMCC).

All polymerase chain reactions (PCR) were carried out on a C1000 Touch ${ }^{\mathrm{TM}}$ thermal cycler (Bio-Rad). DNA sequencing was performed by the Genscript Biotech. Matrix-assisted laser desorption/ionization time-of-flight mass spectrometry (MALDITOF MS) was carried out on Bruker UltraFlextreme. Liquid chromatography electrospray ionization tandem mass spectrometry (LC/ESI-MS/MS) was carried out and processed using a Triple TOF 4600 System (AB SCIEX) equipped with a Prominence Ultra Fast Liquid Chromatography (UFLC) system (Shimadzu). UV-Vis spectrometry was conducted using Cary 300 (Agilent Technologies).

Conditions for all ESI-MS and MS/MS were set as follows: nebulizer gas: 55 psi; heater gas: 55 psi; curtain gas: 35 psi; drying temperature: $550^{\circ} \mathrm{C}$; ion spray voltage: $5500 \mathrm{~V}$; declustering potential: $100 \mathrm{~V}$; collision energy: $35 \mathrm{~V}$ (positive); collision energy spread: $10 \mathrm{~V}$. The mass range and accumulation time are $400-4000 \mathrm{~m} / \mathrm{z}, 250 \mathrm{~ms}$ for ESI-MS and 100-2000 m/z, 100 $\mathrm{ms}$ for MS/MS, respectively. Collision-induced dissociation (CID) was performed for fragmentation of the respective peptide ions. Calibration solutions purchased from AB SCIEX were used for instrument calibration, and high resolution was chosen in the ESI+ mode

338
Production and analysis of thiosparsoamide by S. sparsogenes ATCC 25498.S. sparsogenes ATCC 25498 was spread on PS5 agar plates for sporulation and growth. Approximately $1 \mathrm{~cm}^{2}$ of the agar with sporulated S. sparsogenes ATCC 25498 was used to inoculate $100 \mathrm{~mL}$ of the fermentation medium. After incubation at $28^{\circ} \mathrm{C}$ and $220 \mathrm{rpm}$ for 96 hours, the whole broth was extracted with methanol, lyophilized, and re-dissolved with methanol. The resulting sample was subjected to LCHRMS analysis for thiosparsoamide detection. PS5 agars: $20 \mathrm{~g}$ of starch, $5 \mathrm{~g}$ of pharmamedia, and $20 \mathrm{~g}$ of agar per liter (pH 7.0).Fermentation medium: $25 \mathrm{~g}$ of glucose, $15 \mathrm{~g}$ of soybean meal, $2 \mathrm{~g}$ of dry yeast, and $4 \mathrm{~g}$ of CaCO3 per liter (pH7.0). LCMS analysis of thiosparsoamide was carried out with liquid chromatography heated electrospray ionization tandem mass spectrometry (LC/HESI-MS/MS) and processed using a Thermo Scientific ${ }^{\mathrm{TM}} \mathrm{Q}$ Exactive ${ }^{\mathrm{TM}}$ equipped with a Vanquish Duo UHPLC system (Thermo scienctific). UV-Vis spectrometry was conducted using Diode Array Detector FG. Conditions for HESI-MS and MS/MS were set as follows: sheath gas: 45 psi; auxiliary gas: 15 psi; spare gas: 2.0 psi; capillary temperature: $320{ }^{\circ} \mathrm{C}$; spray voltage: $3500 \mathrm{~V}$; normalized collision energy: $30 \%$ of the available $5 \mathrm{~V}$ (positive); The mass range and accumulation time are $200-2000 \mathrm{~m} / \mathrm{z}, 100 \mathrm{~ms}$ for HESI-MS and data dependent top 20 ions were select by quadrupole selected and HCD (higher collisional dissociation) was performed for fragmentation of the respective ion.

Molecular cloning of spaA, spaF, spaKC, tvaA, tvaF, mutA, mutF, tsdA, tsdF, tsdKC, malA, malF, albA and albF genes. Plasmids containing target genes were synthesized by Genscript Biotech and PCR-amplified by 30 cycles of denaturing ( 95 ${ }^{\circ} \mathrm{C}$ for $30 \mathrm{~s}$ ), annealing $\left(65^{\circ} \mathrm{C}\right.$ for $\left.30 \mathrm{~s}\right)$, and extending $\left(72{ }^{\circ} \mathrm{C}, 1 \mathrm{~min} / \mathrm{kb}\right)$ using high fidelity Phanta DNA Polymerase. Amplifications were confirmed by $2 \%$ agarose gel electrophoresis, and the PCR products were purified using an Omega Biotech Cycle Pure Kit. Target DNA fragments and selected vectors were digested in separate reactions with selected pair of restriction enzymes for $2 \mathrm{~h}$ at $37^{\circ} \mathrm{C}$. The digested products were purified by agarose gel electrophoresis, and the DNA fragments were extracted from the gel using an Omega Biotech Gel Extraction Kit. The resulting DNA products were ligated at $16{ }^{\circ} \mathrm{C}$ for $12 \mathrm{~h}$ in T4 DNA Ligase buffer with T4 DNA Ligase. E. coli DH5 $\alpha$ cells were transformed with $2.5 \mu \mathrm{L}$ of the ligation product by heat shock, and cells were plated on proper antibiotics LB-agar plates and grown for $16 \mathrm{~h}$ at $37^{\circ} \mathrm{C}$. For each plasmid transformant, a single colony was picked to inoculate $5 \mathrm{~mL}$ culture of LB medium with proper antibiotics. The cultures were grown at $37^{\circ} \mathrm{C}$ for $12 \mathrm{~h}$, and plasmids were isolated using an Omega Biotech Plasmid Mini Kit. The sequences of the resulting plasmid products were confirmed by DNA sequencing.

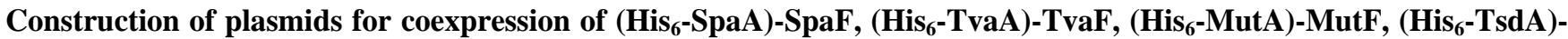
TsdF, ( His $_{6}$-MalA)-MalF and (His ${ }_{6}$-AlbA)-AlbF. Amplification of the two genes was performed using high fidelity Phan- 
$\mathrm{ta}^{\circledR}$ DNA Polymerase following the protocol described above. Restriction digestions were performed with selected pair of restriction enzymes following standard protocol. The digested products of genes were purified by agarose gel electrophoresis and ligated with pRSFDuet- 1 vector. The resulting DNA products were ligated at $16^{\circ} \mathrm{C}$ for $12 \mathrm{~h}$ in $1 \mathrm{X}$ T4 DNA Ligase buffer with T4 DNA Ligase $(0.7 \mathrm{U} / \mu \mathrm{L})$. E. coli DH5 $\alpha$ cells were transformed with $2.5 \mu \mathrm{L}$ of the ligation product by heat shock, and cells were plated on LB-kanamycin agar plates and grown for $16 \mathrm{~h}$ at $37{ }^{\circ} \mathrm{C}$. Several colonies were picked and used to inoculate separate $5 \mathrm{~mL}$ cultures of LB-kanamycin medium. The cultures were grown at $37{ }^{\circ} \mathrm{C}$ for $12 \mathrm{~h}$, and plasmids were isolated using a Omega Biotech Plasmid Mini Kit. The sequences of the resulting plasmid products were confirmed by DNA sequencing.

Mutagenesis of SpaA, SpaF, SpaKC, TsdA and MalA(136) genes. Mutation of SpaA was carried out by 30 cycles of denaturing $\left(95^{\circ} \mathrm{C}\right.$ for $\left.30 \mathrm{~s}\right)$, annealing $\left(65^{\circ} \mathrm{C}\right.$ for $\left.30 \mathrm{~s}\right)$, and extending $\left(72{ }^{\circ} \mathrm{C}, 1 \mathrm{~min} / \mathrm{kb}\right)$ using high fidelity Phanta ${ }^{\circledR}$ DNA Polymerase. Amplifications were confirmed by $1 \%$ agarose gel electrophoresis, and the PCR products were purified using an Omega Biotech Cycle Pure Kit. The target DNA fragment was digested in reaction containing 1X NEB buffer (New England Biolabs) with DpnI for $3 \mathrm{~h}$ at $37^{\circ} \mathrm{C}$. E. coli DH5 $\alpha$ cells were transformed with $10 \mu \mathrm{L}$ of the digested product by heat shock, and cells were plated on LB- kanamycin agar plates and grown for $16 \mathrm{~h}$ at $37^{\circ} \mathrm{C}$. Several colonies were picked and used to inoculate separate $5 \mathrm{~mL}$ cultures of LB-kanamycin medium. The cultures were grown at $37^{\circ} \mathrm{C}$ for $12 \mathrm{~h}$, and plasmids were isolated using an Omega Biotech Plasmid Mini Kit. The sequences of the resulting plasmid products were confirmed by DNA sequencing. The other mutations were carried out by the protocol described above.

Overexpression and purification of SpaF-modified SpaA peptides. E. coli BL21(DE3) cells were transformed with pRS-

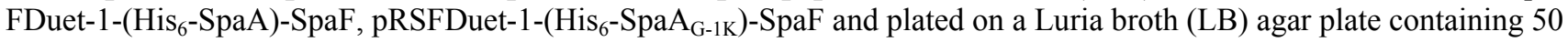
$\mathrm{mg} / \mathrm{L}$ of kanamycin. A single colony was used to inoculate a $5 \mathrm{~mL}$ culture of $\mathrm{LB}$ supplemented with $50 \mathrm{mg} / \mathrm{L}$ of kanamycin. The culture was grown at $37^{\circ} \mathrm{C}$ for $12 \mathrm{~h}$ and was used to inoculate $4 \mathrm{~L}$ of LB containing $50 \mathrm{mg} / \mathrm{L}$ of kanamycin. Cells were grown at $37^{\circ} \mathrm{C}$ to $\mathrm{OD}_{600}=0.6-0.8$ before IPTG was added to a final concentration of $0.2 \mathrm{mM}$ and the culture was incubated at $18{ }^{\circ} \mathrm{C}$ for additional $16 \mathrm{~h}$. Cells were harvested by centrifugation at $12,000 \times \mathrm{g}$ for $25 \mathrm{~min}$ at $4{ }^{\circ} \mathrm{C}$. The resulting cell pellet was resuspended in $30 \mathrm{~mL}$ of start buffer $\left(20 \mathrm{mM} \mathrm{NaH}_{2} \mathrm{PO}_{4}, \mathrm{pH} 7.5,500 \mathrm{mM} \mathrm{NaCl}, 0.5 \mathrm{mM}\right.$ imidazole, $20 \%$ glycerol), and the suspension was sonicated on ice for $20 \mathrm{~min}$ to lyse the cells. Cell debris was removed by centrifugation at $23,700 \times \mathrm{g}$ for $30 \mathrm{~min}$ at $4{ }^{\circ} \mathrm{C}$. The supernatant was discarded, and the pellet containing peptide products was resuspended in $30 \mathrm{~mL}$ of start buffer. The sonication and centrifugation steps were repeated. Again, the supernatant was discarded, and the pellet was resuspended in $30 \mathrm{~mL}$ of buffer 1 ( $6 \mathrm{M}$ guanidine $\mathrm{HCl}, 20 \mathrm{mM} \mathrm{NaH}_{2} \mathrm{PO}_{4}, \mathrm{pH} 7.5,500 \mathrm{mM} \mathrm{NaCl}, 0.5 \mathrm{mM}$ imidazole). The sample was sonicated and insoluble material was removed by centrifugation at $23,700 \times \mathrm{g}$ for $30 \mathrm{~min}$ at $4{ }^{\circ} \mathrm{C}$, followed by filtration of the supernatant through a $0.45 \mu \mathrm{m}$ filter. The filtered sample was applied to a $5 \mathrm{~mL}$ HisTrap HP (GE Healthcare Life Sciences) immobilized metal affinity chromatography (IMAC) column previously charged with $\mathrm{NiSO}_{4}$ and equilibrated in buffer 1. The column was washed with two column volumes of buffer 1, followed by two column volumes of buffer 2 (4 $\mathrm{M}$ guanidine $\mathrm{HCl}, 20 \mathrm{mM} \mathrm{NaH} \mathrm{PO}_{4}, \mathrm{pH} 7.5,500 \mathrm{mM} \mathrm{NaCl}, 30 \mathrm{mM}$ imidazole). The peptide was eluted with 1-2 column volumes of elution buffer ( $4 \mathrm{M}$ guanidine $\mathrm{HCl}, 20 \mathrm{mM} \mathrm{NaH}_{2} \mathrm{PO}_{4}, \mathrm{pH} 7.5,500 \mathrm{mM} \mathrm{NaCl}, 1 \mathrm{M}$ imidazole). The fractions were desalted using a Sep-Pak ${ }^{\circledR}$ C18 Cartridges and analyzed by MALDI-TOF MS and the organic solvents were removed by rotary evaporation, followed by lyophilization. The product was kept at $-80^{\circ} \mathrm{C}$ for long-term storage. Typical yields from 4 $\mathrm{L}$ culture were $1 \mathrm{mg}$ for $\mathrm{His}_{6}$-SpaA peptides.

Overexpression of SpaKC with thioamitide precursor peptides. E. coli BL21(DE3) cells were transformed with pRSFDuet-1-(His ${ }_{6}$-thioamitide precursor peptide) and pACYCDuet-1-SpaKC and plated on a Luria broth (LB) agar plate containing $50 \mathrm{mg} / \mathrm{L}$ of kanamycin and $35 \mathrm{mg} / \mathrm{L}$ of chloramphenicol. A single colony was used to inoculate a $5 \mathrm{~mL}$ culture of LB supplemented with $50 \mathrm{mg} / \mathrm{L}$ of kanamycin and $35 \mathrm{mg} / \mathrm{L}$ of chloramphenicol at $37^{\circ} \mathrm{C}$ for $12 \mathrm{~h}$. The culture was used to inoculate $4 \mathrm{~L}$ of LB containing $50 \mathrm{mg} / \mathrm{L}$ of kanamycin and $35 \mathrm{mg} / \mathrm{L}$ of chloramphenicol. Cells were grown at $37^{\circ} \mathrm{C}$ to $\mathrm{OD}_{600}=0.6$ 0.8 before IPTG was added to a final concentration of $0.2 \mathrm{mM}$ and the culture was incubated at $18{ }^{\circ} \mathrm{C}$ for another $16 \mathrm{~h}$ before harvesting. The purification procedure was described as above.

Overexpression and purification of (SpaKC-SpaF)-modified SpaA peptides. E. coli BL21(DE3) cells were transformed with pRSFDuet-1-His 6 -SpaA-SpaF and pACYCDuet-1-SpaKC or pACYCDuet-1-SpaKC mutants $_{\text {ts }}$ and plated on a Luria broth (LB) agar plate containing $50 \mathrm{mg} / \mathrm{L}$ of kanamycin and $35 \mathrm{mg} / \mathrm{L}$ of chloramphenicol. A single colony was used to inoculate a $5 \mathrm{~mL}$ culture of LB supplemented with $50 \mathrm{mg} / \mathrm{L}$ of kanamycin and $35 \mathrm{mg} / \mathrm{L}$ of chloramphenicol at $37{ }^{\circ} \mathrm{C}$ for $12 \mathrm{~h}$. The cul- 
ture was used to inoculate $4 \mathrm{~L}$ of LB containing $50 \mathrm{mg} / \mathrm{L}$ of kanamycin and $35 \mathrm{mg} / \mathrm{L}$ of chloramphenicol. Cells were grown at $37^{\circ} \mathrm{C}$ to $\mathrm{OD}_{600} \sim 0.6-0.8$ before IPTG was added to a final concentration of $0.2 \mathrm{mM}$ and the culture was incubated at $18{ }^{\circ} \mathrm{C}$ for another $16 \mathrm{~h}$ before harvesting. The purification procedure was described as above.

Overexpression and purification of (SpaKC-LanD $D_{t}$-modified thioamitide precursor peptides. The coexpression of SpaKC, TvaF and TvaA peptide is used as a representative example. E. coli BL21(DE3) cells were transformed with pRSFDuet-1-(His 6 -TvaA)-TvaF and pACYCDuet-1-SpaKC plated on a Luria broth (LB) agar plate containing $50 \mathrm{mg} / \mathrm{L}$ of kanamycin and $35 \mathrm{mg} / \mathrm{L}$ of chloramphenicol. A single colony was used to inoculate a $5 \mathrm{~mL}$ culture of LB supplemented with 50 $\mathrm{mg} / \mathrm{L}$ of kanamycin and $35 \mathrm{mg} / \mathrm{L}$ of chloramphenicol at $37^{\circ} \mathrm{C}$ for $12 \mathrm{~h}$. The culture was used to inoculate $4 \mathrm{~L}$ of LB containing $50 \mathrm{mg} / \mathrm{L}$ of kanamycin and $35 \mathrm{mg} / \mathrm{L}$ of chloramphenicol. Cells were grown at $37^{\circ} \mathrm{C}$ to $\mathrm{OD}_{600}=0.6-0.8$ before IPTG was added to a final concentration of $0.2 \mathrm{mM}$ and the culture was incubated at $18^{\circ} \mathrm{C}$ for another $16 \mathrm{~h}$ before harvesting. The purification procedure was described as above. The other coexpression were carried out by the protocol described above.

Determination of the flavin cofactor bound to SpaF. To identify the type of flavin cofactor, an aliquot of $200 \mu \mathrm{L}$ of a 1.2 $\mathrm{mM}$ solution of $\mathrm{His}_{6}-\mathrm{SpaF}$ was denatured at $100{ }^{\circ} \mathrm{C}$ for $10 \mathrm{~min}$. Precipitated protein was then removed by centrifugation $\left(10,000 \times \mathrm{g}, 10 \mathrm{~min}, 25^{\circ} \mathrm{C}\right)$ and the released flavin was purified by Sep-Pak ${ }^{\circledR} \mathrm{C} 18$ Cartridges and analyzed by LC-ESI-MS.

Modification of SpaA peptides with IAA. To determine the presence of free cysteine, SpaA peptides was digested by trypsin and then incubated with $20 \mathrm{mM} \mathrm{IAA}$ at $37^{\circ} \mathrm{C}$ for $3 \mathrm{~h}$ in $50 \mu \mathrm{L}$ of reaction buffer (50 mM Tris, $\left.\mathrm{pH} 8.6,1 \mathrm{mM} \mathrm{TCEP}\right)$ in the dark, after which the reaction was quenched by addition of $2 \mu \mathrm{L}$ of $1 \mathrm{M}$ DTT to eliminate excess IAA and prevent further side reactions. The reactions were further analyzed by MALDI-TOF.

Reductive desulfurization of modified SpaA peptides. (SpaKC-SpaF)-modified $\mathrm{SpaA}_{\mathrm{G}-1 \mathrm{~K}}(1.0 \mathrm{mg})$ was suspended in 4.0 $\mathrm{mL}$ of $\mathrm{CH}_{3} \mathrm{OH} / \mathrm{H}_{2} \mathrm{O}(1: 1)$, to which $20 \mathrm{mg}$ of $\mathrm{NiCl}_{2}$ and $20 \mathrm{mg}$ of $\mathrm{NaBH}_{4}$ were added. This mixture was stirred under $1 \mathrm{~atm}$ of $\mathrm{H}_{2}$ at room temperature. After $8.5 \mathrm{~h}$, the mixture was taken off the hydrogenation apparatus, and then centrifuged, followed by the removal of the supernatant. Mixed solvent of $\mathrm{CH}_{3} \mathrm{OH} / \mathrm{H}_{2} \mathrm{O}$ (ration=1:1, 2.0 mL) was added to the black nickel boride pellets, and the suspension was sonicated for 15 minutes. The suspension was centrifuged, and the supernatants were collected. The organic solvent was removed in vacuo, and the desulfurized peptide was treated with trypsin. Digestion conditions: $20 \mathrm{mM}$ Tris- $\mathrm{HCl}, \mathrm{pH}=8.0,1 \mu \mathrm{M}$ trypsin, $100 \mu \mathrm{M}$ peptide product at $37^{\circ} \mathrm{C}$ for $6 \mathrm{~h}$.

Measurement of protein-protein and protein-peptide interactions by MST assays. As a representative example, the binding affinity between $\mathrm{SpaKC}$ and $\mathrm{SpaA}_{\text {leader }}$ was measured using Monolith NT.115 Pico (Nanotemper Technologies). SpaKC was fluorescently labelled by the labeling kit (Monolith Protein Labeling Kit RED-NHS 2nd Generation (Amine Reactive), MO-L011). A volume of $90 \mu \mathrm{L}$ SpaKC sample $(10 \mu \mathrm{M})$ in the labelling buffer $(130 \mathrm{mM} \mathrm{NaHCO} 3,50 \mathrm{mM} \mathrm{NaCl}, \mathrm{pH}$ 8.2) was mixed with $10 \mu \mathrm{L}$ dye (Dye RED-NHS 2nd Generation (Nanotemper MO-L011)) solution (300 $\mu \mathrm{M})$ for $30 \mathrm{~min}$ at room temperature in the dark. Next, the SpaKC sample was loaded to column B (Nanotemper MO-L011) and eluted with $450 \mu \mathrm{L}$ of assay buffer $\left(50 \mathrm{mM} \mathrm{Na}_{2} \mathrm{CO}_{3} / \mathrm{NaHCO}_{3}, 150 \mathrm{mM} \mathrm{NaCl}, 10 \mathrm{mM} \mathrm{MgCl}_{2}, 0.07 \%\right.$ tween $\left.20, \mathrm{pH} 9.0\right)$. The labelled SpaKC sample was diluted until pretest fluorescence excitation range from 16000-20000 for the most optimized response $(\sim 1$ $\mathrm{nM})$. To perform the MST assay, the labelled SpaKC sample $(10 \mu \mathrm{L})$ was first incubated with SpaA peptide $(10 \mu \mathrm{L})$ of 16 different serial dilutions in the assay buffer for $5 \mathrm{~min}$ to allow binding. The samples were then loaded into Monolith NT.115 capillary (Nanotemper Technologies) and measured by using 20\% (Auto-detect) Pico - RED as excitation power and medium MST power. The binding measurement was repeated three times. Data analysis was performed using Nanotemper affinity analysis software.

To measure the binding affinity between SpaKC-SpaF-SpaA $\mathrm{A}_{\text {leader }}$, SpaF was incubated separately with $\mathrm{SpaA}_{\text {leader }}$ peptide of 16 different serial concentrations in assay buffer. The fluorescent-labelled SpaKC $(10 \mu \mathrm{L})$ was mixed with these 16 samples and incubated for $5 \mathrm{~min}$ before loading into Monolith NT.115 capillary. The measurement condition and data analysis were the same as SpaKC-SpaAleader binding affinity measurement mentioned before. 
468 In vitro modification of $\mathrm{SpaA}_{\mathrm{G}-1 \mathrm{~K}}$ by SpaKC, SpaF and SpaKC-SpaF combination. Typically, SpaA peptide $(25 \mu \mathrm{M})$

469 was incubated with SpaKC, SpaF or SpaKC-SpaF $(12.5 \mu \mathrm{M})$ in reaction buffer $\left(500 \mathrm{mM} \mathrm{Na} \mathrm{CO}_{3} / \mathrm{NaHCO}_{3}, 200 \mathrm{mM} \mathrm{NaCl}\right.$, $4701 \mathrm{mM}$ TCEP, $\mathrm{pH} 9.0$ ). $5 \mathrm{mM}$ ATP and $5 \mathrm{mM} \mathrm{MgCl}_{2}$ were supplied when SpaKC is present. All assays were carried out at 28 $471{ }^{\circ} \mathrm{C}$ for $4 \mathrm{~h}$ before quenched with $1 \%$ formic acid. The supernatant was lyophilized, re-dissolved in $\mathrm{H}_{2} \mathrm{O}$ and desalted by a SPE 472 column. The resulting sample was lyophilized, re-dissolved and digested by trypsin before analyzed by LC-MS/MS. 